\title{
Usefulness of the blood chemistry screening associated with DKA imaging in a case of secondary osteoporosis in a young adult
}

\begin{abstract}
Multiple myeloma is rare in young patients and may be undiagnosed or characterized by a significant delay in diagnosis.

We report the association of stage I myeloma (according to Durie-Salmon classification) and osteoporosis in a 37-year-old male patient complaining of worsening pain in the spine. Pain attacks resulted in increases in blood pressure with hypertensive episodes. The investigations (on the basis of an unclear radiological picture and the presence of hypertensive state) led to the diagnosis of low grade osteoporosis. Since the young subject was symptomatic at the diagnosis, we continued evaluations on the basis of imaging data (DXA) and laboratory tests, revealing the presence of multiple myeloma. Clinical, laboratory, and therapeutic implications of this clinical case are discussed.
\end{abstract}

Keywords: Osteoporosis; Young Patient; Hematologic Tests; Multiple Myeloma Utilità dello screening ematochimico associato a DXA in un caso di osteoporosi secondaria dell'adulto

CMI 2017; 11(1): 45-48

https://doi.org/10.7175/cmi.v11i1.1288

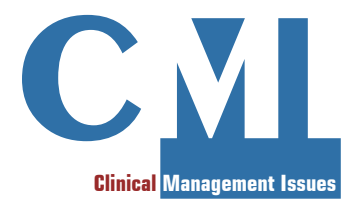

Case report
Department of Internal Medicine and Geriatrics, University of Campania "Luigi Vanvitelli", Naples

Geriatrician, ASL

NA1 Centro, Naples

3 Medical Director and Geriatrician Manager at the Center Home Care (CAD), ASL Roma 2, Rome

\section{INTRODUCTION}

Multiple myeloma is rare under the age of 30 (less than $0.3 \%$ ) and may be undiagnosed or characterized by a significant delay in diagnosis, which usually occurs at the first event of fracture [1]. Moreover, treatment outcomes in younger adults are better than those observed in older patient groups, thus confirming the importance of early detection in these pathologies.

The current guidelines recommend the use of both imaging (Dual-energy X-ray absorptiometry, DXA) and laboratory tests, in order to evaluate various conditions, among which osteoporosis is the common denominator. The minimum cost-effective group of tests to be performed in order to investigate the presence of occult metabolic disorders in adults at risk of falls and fractures or in case of suspected osteoporosis should include erythrocyte sedimentation rate, complete blood count, complete and fractional proteinemia, serum calcium and urinary calcium, phosphorus, creatinine (with estimated glomerular filtration rate-eGFR), and alkaline phosphatase. However, second-level laboratory tests are indicated when in firstlevel exams the clinical picture is associated

Why we describe this case

The described case report highlights the issue of evaluation of secondary osteoporosis and osteoporosis in young patients, because the complications of these cases, too often misunderstood until the fracture event, can be serious and the prognosis is significantly related to the delay in the diagnosis
Corresponding author Dr. Valerio Massimo Magro Department of Internal Medicine and Geriatrics, University of Campanis "Luigi Vanvitelli", Piazza L. Miraglia 2, 80100, Naples, Italy Tel: 3492224922 valerio_magro@hotmail.com

Disclosure

The Authors declare that they have no conflict of financial interest in the topics covered in this article 


\begin{tabular}{|c|c|c|c|c|}
\hline Parameter & $\begin{array}{l}\text { Detected } \\
\text { Ievel (g/dil) }\end{array}$ & $\begin{array}{l}\text { Normal } \\
\text { Ievels (g/dil) }\end{array}$ & $\begin{array}{l}\text { Detected } \\
\text { Ievel }(\%)\end{array}$ & $\begin{array}{l}\text { Normal } \\
\text { Ievels }(\%)\end{array}$ \\
\hline Albumin & 4.7 & $3.5-5$ & 56.8 & $55.8-66.1$ \\
\hline a1-globulins & 0.23 & $0.2-0.4$ & 3.5 & $2.9-4.9$ \\
\hline a2-globulins & 0.63 & $0.4-0.8$ & 9.8 & 7.1-11.8 \\
\hline$\beta 1$-globulins & 0.43 & $0.6-1$ & 6 & $4.7-7.2$ \\
\hline$\beta 2$-globulins & 1.15 & $0.6-1$ & 14.3 & $3.2-6.5$ \\
\hline$\gamma$-globulins & 0.70 & $0.9-1.4$ & 9.6 & $11.1-18.8$ \\
\hline Total proteins & 7.6 & - & - & - \\
\hline A/G ratio & 1.43 & $1.10-2.40$ & - & - \\
\hline
\end{tabular}

Table I. Results about proteins in the blood tests (percentage and absolute levels)

$\mathrm{A} / \mathrm{G}$ ratio $=$ total protein and albumin/globulin ratio

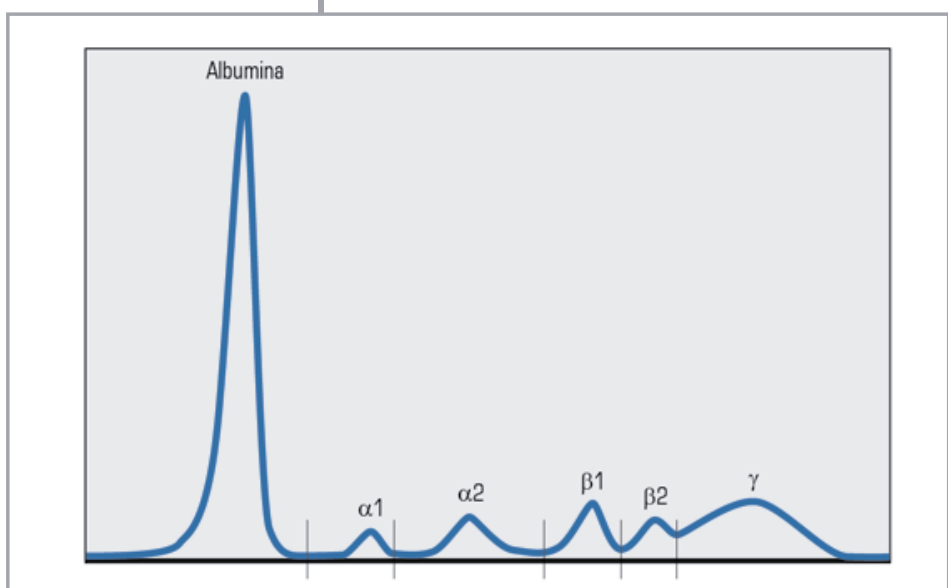

Figure 1.

Electrophoretic test showing an electrophoretic pattern of proteins with anomalies with abnormalities possibly suggestive of the disease: ionized calcium, thyroid-stimulating hormone- $\mathrm{TSH}$, serum parathyroid hormone-PTH, 25-OH-vitamin D, dexamethasone suppression test, total testosterone (in men), serum and urine immunofixation, serum ferritin, anti-transglutaminase antibodies, and serum tryptase levels [2]. Although secondary osteoporosis accounts for a small minority $(<5 \%)$ of the total cases of osteoporosis in women, it represents an important cause of disease in male patients, and may indicate the presence a serious illness, sometimes with poor prognosis (especially in case of delayed diagnosis).

In this case report, the assessment of musculoskeletal and cardiovascular systems in a young male patient led to an early diagnosis of a potentially fatal disease.

\section{CASE PRESENTATION}

A 37-year-old patient, construction worker, came to our hospital for pressure control and complaining about a pain localized in the dorsal and lumbar spine. Vital signs were within limits, except blood pressure$\mathrm{BP}=140 / 100 \mathrm{mmHg}$. Pain was present on percussion of the spinous processes from $5^{\text {th }}$ to $7^{\text {th }}$, with a mild paresthesia in the right upper limb, reported to be present for many years. His recent blood count, thyroid function, renal, and lipid profile tests were all normal.

24-hour monitoring of the blood pressure showed "daytime diastolic blood pressure at upper limit of normal and occasional nighttime systolic and diastolic blood pressure levels slightly exceeding the limits, with preserved circadian rhythmicity". Chest $\mathrm{X}$-ray revealed a right-convex scoliosis, in the absence of pulmonary foci or pleural abnormalities. The patient started antihypertensive therapy (ACE-inhibitor) and a regimen of NSAIDs, with poor blood pressure control and pain (Number Rating Scale-NRS = 4/10).

A new lumbar spine and pelvis X-ray showed hyperlucency of the lumbar spine with unscathed pelvis and a mild osteoporosis framework, as evidenced by another DXA (the exam was performed privately, with a T-score $=-2.6$ at the level of L2 vertebral body and a total $\mathrm{T}$-score at the spine $=-2.0$ ). The radiological image did not show any vertebral fracture. Analyzing the morphometry of the vertebral bodies, no significant decrease (as to fall within the definition of fracture) in the height of the vertebrae was detected (semi-quantitative method of H. K. Genant). At this stage, $1^{\text {th }}$ level blood tests were performed, showing normal levels, except the percentage of $\beta 2$-globulins and $\gamma$-globulins, as reported in Table I and Figure 1.

Owing to the suspected monoclonality in $\beta 2$ zone, immunofixation and other blood tests were performed, showing the results reported in Table II.

$\kappa$ chains were absent, while the $\lambda$ chains were present $(3,67 \mathrm{~g} / 1$; normal values: 0.90 2.10; Figure 2).

A fine needle aspiration of bone marrow was performed, finding plasma cells infiltrate in $15 \%$ of the examined bone marrow. Multiple myeloma was staged according to Durie-Salmon staging and the New International Staging System (stage 1). The patient was taken in charge by a multidisciplinary team formed by hematologists and orthopedists to carry on the diagnostic (scintigraphy) and therapeutic management (careful observation with seriated controls over time; possible preventive osteosynthesis), while we advised to start therapy with bisphosphonates. 


\section{DISCUSSION}

Multiple myeloma is a malignant tumor arising from plasma cells, frequently with bone involvement (including vertebral bodies and pelvis). Solitary lesions without systemic involvement may remain silent for many years, and often diagnosis is occasional: in this case, our diagnosis was due to hypertension (hyperviscosity?) and algic issues, the latter being difficult to investigate, because of the patient's scoliosis and work history.

The electrophoretic test (among the routine tests for the evaluation of osteoporosis) put in evidence a peak with migration (seen at lower frequency) in the $\beta$ zone, with a $\operatorname{IgA}$ peak in the pre- $\gamma$-area. Generally, altered immunoglobulins tend to associate with alterations in calcium metabolism: hypercalcemia, which is absent in this case, is found in about $30-40 \%$ of patients. Perhaps, our patient was the bearer of a previously unidentified and unstudied or unfollowed monoclonal gammopathy of uncertain significance (MGUS) over time, already with initial bone damage (but the patient was not able to report tests or previous diagnosis of this condition) $[3,4]$. The pain after spine percussion was an important warning signal that led to a deepening of diagnostic investigation. However, back pain is a common sign and fortunately most cases are due to benign causes. Anyway, serious causes such as cancer, infection, and even fractures must been considered [5]. As in the scenarios described in the literature, unexplained changes in bone mineral density - as in the present case - may trigger a more extensive evaluation, including the search for monoclonal components [6].

\section{CONCLUSIONS}

Unquestionably osteoporosis in young individuals is less frequent than in adults and the elderly. But in this population group, the development of osteoporosis is often associated with secondary causes $[7,8]$. In fact, almost $50 \%$ of young people with osteoporosis have diseases or therapies related to the development of this disorder.

In order to rule out secondary causes of osteoporosis, laboratory studies should be extensive. Therefore, it is important to perform blood chemistry tests in all patients with osteoporosis or a recent clinical fracture. If necessary, additional tests can be performed.

National and international guidelines are focused on the diagnosis of secondary os-

\begin{tabular}{|c|c|c|}
\hline Parameter & Detected level & Normal levels \\
\hline $\lg A(\mathrm{mg} / \mathrm{d} \mid)$ & 1144 & $63-484$ \\
\hline $\lg G(\mathrm{mg} / \mathrm{dl})$ & 727 & 540-1822 \\
\hline $\lg M(\mathrm{mg} / \mathrm{d})$ & 73 & $33-293$ \\
\hline$\beta 2$-microglobulin (mg/l) & 2.32 & $0.97-2.64$ \\
\hline $\begin{array}{l}\text { Total serum calcium } \\
\text { concentration (mg/dl) }\end{array}$ & 9 (correct calcium 8.79) & $8.5-10.5$ \\
\hline
\end{tabular}

teoporosis in the field of differential diagnosis with postmenopausal and senile forms $[9,10]$. Recommendations are less accurate Table II. Results from immunofixation and other blood tests about osteoporosis in the youth and even more so about secondary causes of osteoporosis in young patients.

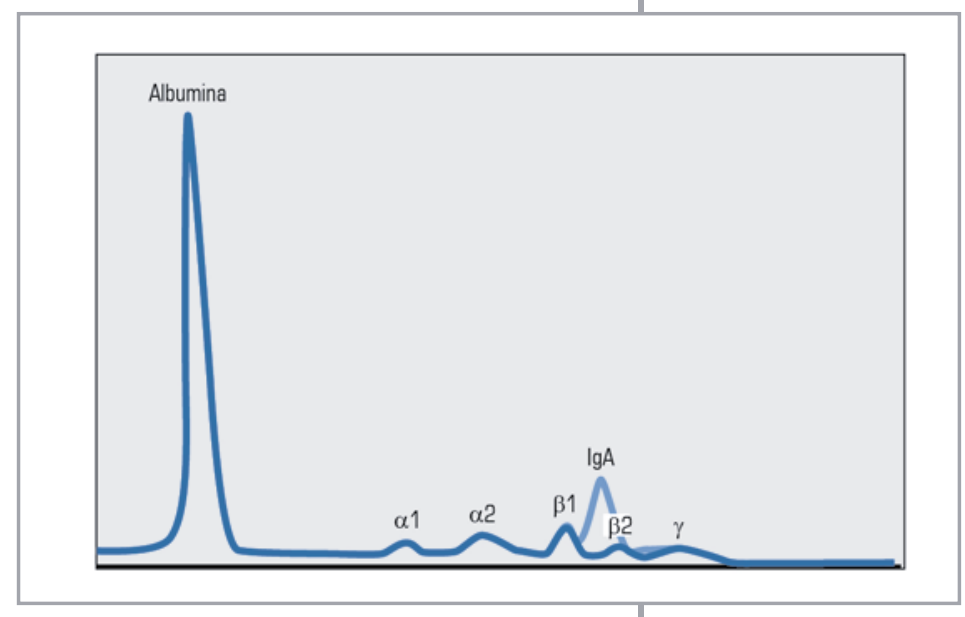

Considering that prevention plays a key role in reducing the consequences of serious diseases, the laboratory plays an even more important role. Laboratory studies may show potentially reversible abnormalities that must be assessed and corrected, if possible, before starting pharmacological therapy, or silent secondary osteoporosis, requiring other types of treatment. In fact, secondary osteoporosis has a different etiology and can evolve asymptomatically until obvious signs of fracture or osteoporosis emerge, so that many subjects in the young adult age or adult but not elderly individuals may escape the assessments because for a long time they are considered healthy subjects, at least until the time of the first event of fracture $[11,12]$. Therefore, the prompt recognition of secondary osteoporosis may have significant repercussions both on the patient's quality of life and the quoad vitam prognosis.
Figure 2. Results from electrophoretic test, bighlighting an increase in immunoglobulin $A$ 


\section{Keypoints}

- The presence of pain on percussion of spinous processes should be a warning signal even in young patients with predisposing factors to back pain

- Secondary osteoporosis has a different etiology as compared to primary osteoporosis, and can evolve asymptomatically until the first event of fracture

- Laboratory studies play a key role in the diagnosis

- Secondary osteoporosis may be due to multiple myeloma

- Multiple myeloma is rare under the age of 30 and may be undiagnosed or characterized by a significant delay in diagnosis. A timely diagnosis is crucial in this life-threatening disease

\section{REFERENCES}

1. Jurczyszyn A, Nahi H, Avivi I, et al. Characteristics and outcomes of patients with multiple myeloma aged 21-40 years versus 41-60 years: a multi-institutional case-control study. $\mathrm{BrJ}$ Haematol 2016; 175: 884-91; https://doi.org/10.1111/bjh.14328

2. Mirza F, Canalis E. Management of endocrine disease: Secondary osteoporosis: pathophysiology and management. Eur J Endocrinol 2015; 173: R131-R151; https://doi.org/10.1530/EJE-150118

3. Drake MT. Unveiling skeletal fragility in patients diagnosed with MGUS: no longer a condition of undetermined significance? J Bone Miner Res 2014; 29: 2529-33; https://doi.org/10.1002/ jbmr.2387

4. Murray DL, Seningen JL, Dispenzieri A, et al. Laboratory persistence and clinical progression of small monoclonal abnormalities. Am J Clin Pathol 2012; 138: 609-13; https://doi.org/10.1309/ AJCPT6OWWMHITA1Y

5. Dugan LO, Dugan DA, Dugan WM Jr. Back pain: the primrose path - a case report. Indiana Med 1990; 83: 114-6

6. Faiman B, Licata AA. New tools for detecting occult monoclonal gammopathy, a cause of secondary osteoporosis. Cleve Clin J Med 2010; 77: 273-8; https://doi.org/10.3949/ ccjm.77a.09091

7. Bours SP, van den Bergh JP, van Geel TA, et al. Secondary osteoporosis and metabolic bone disease in patients 50 years and older with osteoporosis or with a recent clinical fracture: a clinical perspective. Curr Opin Rheumatol 2014; 26: 430-9; https://doi.org/10.1097/ BOR.0000000000000074

8. Bours SP, van GeelTA, Geusens PP, et al. Contributors to secondary osteoporosis and metabolic bone diseases in patients presenting with a clinical fracture.J Clin Endocrinol Metab 2011; 96 : 1360-7; https://doi.org/10.1210/jc.2010-2135

9. Camacho PM, Petak SM, Binkley N, et al. American Association of Clinical Endocrinologists and American College of Endocrinology Clinical Practice Guidelines for the diagnosis and treatment of postmenopausal osteoporosis - 2016 - Executive summary. Endocr Pract 2016; 22 (Suppl 4): 1-42; https://doi.org/10.4158/EP161435.GL; https://doi.org/10.4158/EP161435. ESGL; https://doi.org/10.4158/EP161208.GL

10. Adami S, Bertoldo F, Brandi ML, et al. Guidelines for the diagnosis, prevention and treatment of osteoporosis. Reumatismo 2009; 61: 1-25

11. Harrington J, Sochett E. The child with multiple fractures, what next? Pediatr Clin North Am 2015; 62: 841-55; https://doi.org/10.1016/j.pcl.2015.04.006

12. Järveläinen H, Remes K, Viikari J. A severe osteoporosis in a middle-aged man. Duodecim 1999; 115: 2473-6 